\title{
Trifluorothymidine versus adenine arabinoside in the treatment of herpes simplex keratitis
}

\author{
O. P. VAN BIJSTER VELD AND H. POST \\ From the Koninklijk Nederlands Gasthuis voor Ooglijders, F.C. Dondersstraat 65, Utrecht, Holland
}

SUMMARY Trifluorothymidine (TFT) and adenine arabinoside (ara-A) are effective antiviral drugs with a very low toxicity for the cornea. In our study no difference between these 2 drugs in antiviral activity was noted. The average healing time for TFT was $11 \cdot 14$ days and for ara-A 10.54 days. These data differ markedly from those of other studies. This was the result of the use of additional criteria for healing of the lesion. Not only absence of fluorescein staining of the cornea but also the absence of oedema and cystic changes in the epithelium over the previous ulcer were considered criteria for healing. In addition to clearly defined healing criteria and the healing time we found the interval between the first symptoms and the commencement of the therapy of greatest importance in the clinical evaluation of antiviral drug efficacy. An effort was made to approximate this relationship for TFT and ara-A mathematically.

The introduction of idoxuridine (IDU) in 1962 (Kaufman et al., 1962) was the first rational approach to the treatment of dendritic keratitis. Laibson and Leopold (1964), however, found the effectiveness of IDU to be no greater than that of curettage of the corneal epithelium. Moreover, Jones (1967) found IDU to be toxic when used over a long period, and this is a common experience.

In 1972 Wellings et al. (1972) published their results with a new antiviral drug, trifluorothymidine (TFT), and found it to be more effective than IDU. In that trial no toxic side effects were noted. In the same year Pavan-Langston and Dohlman (1972) reported that they found another antiviral drug, adenine arabinoside (ara-A), also to be more effective than IDU.

Coster et al. (1976) studied the healing time and the rate of healing of superficial herpetic keratitis treated with trifluorothymidine and adenine arabinoside. These workers were unable to find any difference in the healing time of TFT and ara-A with regard to dendritic keratitis. For amoeboid ulcers, however, they found TFT to be possibly more effective than ara-A.

We have reinvestigated the efficacy of trifluorothymidine and that of adenine arabinoside in 56 cases of dendritic keratitis, paying special attention

Correspondence to Dr O. P. van Bijsterveld, Koninklijk Nederlands Gasthuis voor Ooglijders, F.C. Dondersstraat 65, Utrecht, Holland. to the influence of the length of the interval between the appearance of the first symptoms of the disease and the beginning of treatment and to the age of the patient on the healing time. The investigation was done in a double-blind comparative trial.

\section{Materials and methods}

Of a total of 66 patients ( 43 males and 23 females) with dendritic keratitis, 36 males and 20 females were studied. Three male patients were excluded from the study because of omissions in complying with the examination protocol. An additional 7 patients were excluded from the study because their ulcers failed to heal within 23 days, which was our arbitrary limit. Patients who received steroid medication were excluded from the trial.

Of the patients included 17 males and 11 females were treated with trifluorothymidine and 19 males and 9 females with adenine arabinoside. Treatment was given on an outpatient basis. Each patient received a coded treatment sequentially allocated by the central dispensing unit. The preparations consisted of either $2 \%$ trifluorothymidine ointment or $3 \%$ adenine arabinoside ointment, both specially prepared for this trial. The ointment tubes were identical in design.

The coded antiviral ointment was given according to the following specifications: Every 4 hours 4 to $5 \mathrm{~mm}$ of ointment was to be placed in the lower cul de sac, the first application to be given early in the 
morning, the last at bedtime. In addition scopolamine eyedrops $0.2 \%$ were given, usually once or twice daily.

When the epithelial defect of the dendritic keratitis had healed, the treatment was continued for one more week. Healing was defined as the situation in which no staining with fluorescein was observed and no epithelial oedema and cystic changes were present in the epithelium covering the site of the original ulcer. Treatment was discontinued after 22 days. Cases that were not healed after 22 days were not included in the analysis and were considered to be failures. Data were recorded on a history and examination sheet. Follow-up examinations were done with special reference to the condition of the anterior segment of the eye. Fluorescein and Bengal rose staining were done to judge the condition of the epithelium.

\section{Results}

Seven out of 63 treated ulcers failed to heal within 23 days, which was our arbitrary limit. These cases were excluded from the analysis. Four of these patients were treated with ara-A and three with TFT. In all these patients the ulcer was initially closed as judged by fluorescein examination, but the epithelium in these cases showed areas of oedema and cystic changes in the area of the healed ulcer. The average number of days it took these cases to heal was 32 days, with a minimum of 23 and a maximum of 54 days. These therapeutic failures represented cases of metaherpes complicating the dendritic ulcers. After clinical recurrence of infection, stromal lesions developed eventually.

Table 1 Healing time for dendritic ulcers arranged according to treatment, sex, and eye

\begin{tabular}{|c|c|c|c|c|c|c|c|c|}
\hline & \multicolumn{4}{|l|}{$T F T$} & \multicolumn{4}{|l|}{ ara- $A$} \\
\hline & \multicolumn{2}{|c|}{ Male } & \multicolumn{2}{|c|}{ Female } & \multicolumn{2}{|l|}{ Male } & \multicolumn{2}{|c|}{ Female } \\
\hline & $O D$ & $O S$ & $O D$ & $O S$ & $O D$ & $o s$ & $O D$ & $O S$ \\
\hline & 3 & 21 & 21 & 4 & 7 & 21 & 7 & 20 \\
\hline & 8 & 12 & 7 & 13 & 7 & 7 & 2 & 9 \\
\hline & 12 & 16 & 5 & 18 & 12 & 9 & 6 & 13 \\
\hline & 8 & 4 & 6 & 12 & 22 & 7 & 4 & 14 \\
\hline & 13 & 9 & & 20 & 14 & 8 & & 5 \\
\hline & 16 & 14 & & 16 & 16 & 9 & & \\
\hline & 8 & 5 & & 6 & 12 & 14 & & \\
\hline & 10 & & & & & 14 & & \\
\hline & 13 & & & & & 11 & & \\
\hline & 12 & & & & & 14 & & \\
\hline & & & & & & 5 & & \\
\hline & & & & & & 6 & & \\
\hline Mean & $10 \cdot 3$ & $11 \cdot 6$ & $9 \cdot 75$ & $12 \cdot 71$ & $12 \cdot 86$ & 10.42 & $4 \cdot 75$ & $12 \cdot 2$ \\
\hline
\end{tabular}

Table 2 Analysis of main effects: therapy, sex, and eye and their interactions. Analysis of variance

\begin{tabular}{|c|c|c|c|c|}
\hline $\begin{array}{l}\text { Nature of } \\
\text { effect }\end{array}$ & Source & $\begin{array}{l}\text { Sum of } \\
\text { squares }\end{array}$ & $D F$ & $\begin{array}{l}\text { Mean sum } \\
\text { of squares }\end{array}$ \\
\hline \multirow[t]{3}{*}{ Main factors } & Therapy (Th) & $5 \cdot 1607$ & 1 & $5 \cdot 1607$ \\
\hline & Sex $\quad$ (S) & 6.0036 & 1 & 6.0036 \\
\hline & Eye (E) & 28.8517 & 1 & $28 \cdot 8517$ \\
\hline Interactions & $\mathbf{T h} \times \mathbf{S}$ & $34 \cdot 3790$ & 1 & $34 \cdot 3790$ \\
\hline between pairs & $T h \times E$ & $6 \cdot 2623$ & 1 & $6 \cdot 2623$ \\
\hline of factors & $\mathbf{S} \times \mathbf{E}$ & $105 \cdot 7896$ & 1 & $105 \cdot 7896$ \\
\hline Interactions & & & & \\
\hline $\begin{array}{l}\text { between triplets } \\
\text { of factors }\end{array}$ & $\mathbf{T h} \times \mathbf{S} \times \mathbf{E}$ & $37 \cdot 7900$ & 1 & $37 \cdot 7900$ \\
\hline Residual & & $1267 \cdot 3167$ & 48 & $26 \cdot 4024$ \\
\hline Total & & $1491 \cdot 5536$ & 55 & \\
\hline
\end{tabular}

$\mathrm{DF}=$ degrees of freedom.

$F_{48}^{1}=4.04 ; \alpha=0.05$. Note the $F$ value of sex $\times$ eye interaction $=F_{48}^{1}$ $=4.0068$, which is almost significant.

In Table 1 the number of days the dendritic ulcers took to heal are given. The data are classified according to type of treatment, sex, and eye. The average number of days for the dendritic ulcer to heal in the TFT-treated group was $11 \cdot 14$ days and in the ara-A-treated group 10.54 days. The average healing time for males was 11.08 and for females 10.40 days. For the right eyes the ulcers took 10.04 and for the left eyes 11.48 days to heal. None of these differences were statistically significant.

In Table 2 an analysis of variance is given. None of the tested factors or interactions are statistically significant. The sex $\times$ eye interaction is noteworthy; the $F$ ratio with 1 and 48 degrees of freedom at the $5 \%$ level is 4.04 . The $F$ ratio of the sex $\times$ eye interaction is 4.01 and therefore almost significant at that level. This would imply that herpetic ulcers in the right eye of women would heal more rapidly; this does not make sense. Reviewing the histories of the patients in this category we found that these women received treatment at a very early stage of the disease.

In Fig. 1 the scatter diagram of the relationship between the number of days that elapsed between the appearance of the first symptoms of the disease and the moment treatment was initiated and the number of days it took the ulcer to heal is given for the TFT and ara-A treated groups combined. Correlation analysis shows a highly significant relationship between these 2 quantities. If the TFT group is analysed separately, there is no significant correlation. This was the result of some extremely long healing periods in patients who were treated early. They were 2 patients who developed interstitial infiltrations. Furthermore, these patients received treatment on the fifth day and had ex- 


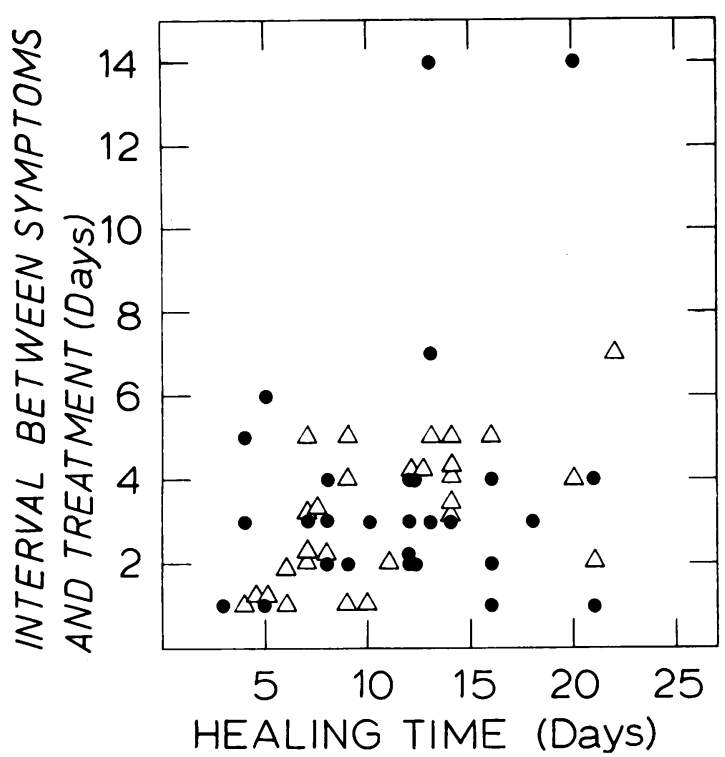

Fig. 1 The relationship between the interval between appearance of first symptoms and initiation of the treatment and the healing time: TFT $(O)$ and ara- $A(\triangle)$

tremely short healing times. They had a very mild stellate type of herpes keratitis. The healing times of these patients are marked with an asterisk in Table 3.

In Fig. 2 the type of association is shown graphically. It represents the mathematical approximation of the type of association between the interval before treatment and the average healing time of both groups combined. A second-degree curve defined by the formula $y=+11.88+(2.298 \times$ $\left.-0.485 \mathrm{x}^{2}\right)$, with origin at day 3 and $\mathrm{x}$-unit equals 1 day, appears to be a good fit.

With regard to the interval before treatment and healing time, the TFT and ara-A group did not differ significantly (Table 4). In Table 5 the importance of very early treatment is shown.

No significant correlation was found between the age of the patients and healing time. Also no statistically significant difference existed in the TFT and ara-A treated groups with regard to age. The average age in the TFT-treated group was $45 \cdot 18$ (33.13-57.23, 99\% confidence limits) and for the ara-A-treated group this was 46.14 (33.25-59.02, $99 \%$ confidence limits).

In 5 out of 17 patients that received the virostatic treatment longer than 14 days a very mild diffuse epithelial staining was observed with Bengal rose.

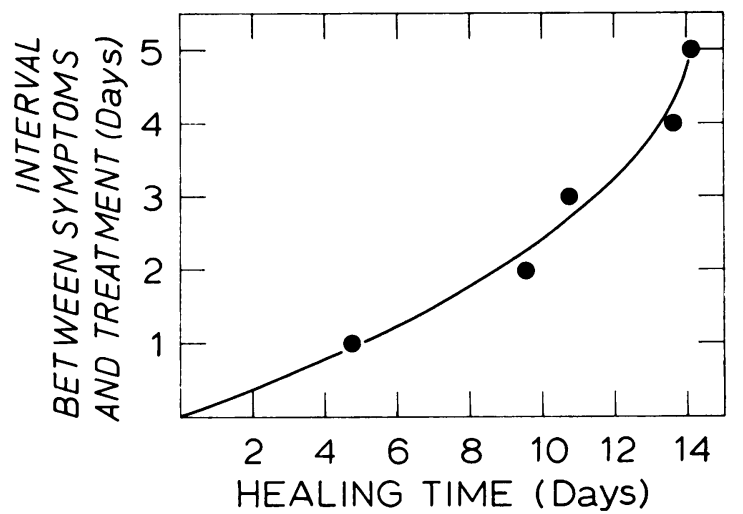

Fig. 2 A second degree curve is a good approximation to the type of relationship between the interval before treatment and healing time

Table 3 The relationship of the interval in days between beginning of symptoms and the treatment, and the healing time of the dendritic ulcer. The figures marked with an asterisk differ very noticeably from the general averages

\begin{tabular}{|c|c|c|c|c|c|c|c|c|c|c|}
\hline \multirow{3}{*}{$\begin{array}{l}\text { Healing } \\
\text { time in } \\
\text { days }\end{array}$} & \multicolumn{10}{|c|}{ Interval between beginning of symptoms and treatment in days } \\
\hline & \multicolumn{2}{|l|}{1} & \multicolumn{2}{|l|}{2} & \multicolumn{2}{|l|}{3} & \multicolumn{2}{|l|}{4} & \multicolumn{2}{|l|}{5} \\
\hline & $T F T$ & ara- $A$ & $T F T$ & $\operatorname{ara}-A$ & $T F T$ & $a r a-A$ & $T F T$ & ara $-A$ & $T F T$ & ara $-A$ \\
\hline & $21 *$ & 9 & 16 & 21 & 7 & 7 & 21 & 20 & 4* & 7 \\
\hline & 3 & 2 & 12 & 7 & 8 & 7 & 12 & 12 & 13 & 9 \\
\hline & $16^{*}$ & 5 & 9 & 7 & 18 & 14 & 8 & 9 & $5 *$ & 22 \\
\hline & 5 & 5 & 8 & 8 & 4 & 14 & 12 & 14 & 20 & 13 \\
\hline & & 4 & 6 & 11 & 13 & & 16 & 14 & 13 & 14 \\
\hline & & & 6 & 6 & 14 & & & 12 & & 16 \\
\hline & & & & 6 & 10 & & & & & \\
\hline & & & & & 12 & & & & & \\
\hline Mean & $11 \cdot 25$ & 5.00 & $9 \cdot 5$ & $9 \cdot 4$ & $10 \cdot 75$ & $10 \cdot 5$ & $13 \cdot 8$ & $13 \cdot 5$ & 11.0 & $13 \cdot 5$ \\
\hline Number & 4 & 5 & 6 & 7 & 8 & 4 & 5 & 6 & 5 & 6 \\
\hline SD & $8 \cdot 6$ & $2 \cdot 5$ & 3.9 & 5.4 & 4.4 & $4 \cdot 04$ & 4.9 & 3.7 & 6.6 & $5 \cdot 3$ \\
\hline
\end{tabular}


Table 4 Analysis of variance of the interval between the beginning of the symptoms and of treatment in the TFT and ara- $A$ treated groups

\begin{tabular}{lrrl}
\hline $\begin{array}{l}\text { Source of } \\
\text { variation }\end{array}$ & $\begin{array}{l}\text { Sum of } \\
\text { squares }\end{array}$ & DF & $\begin{array}{l}\text { Mean sum of } \\
\text { squares }\end{array}$ \\
\hline Between treatments & $7 \cdot 1420$ & 1 & $7 \cdot 1429$ \\
Within treatments & 352.5714 & 54 & 6.5291 \\
Total & $359 \cdot 7134$ & 55 & \\
\hline
\end{tabular}

$F_{54}^{1}=1.09 ;$ not significant.

Table 5 Differences in healing time and percentage gain if treatment were started 24 hours earlier than stated day

\begin{tabular}{lll}
\hline Day & $\begin{array}{l}\text { Average healing time } \\
\text { in days }\end{array}$ & $\begin{array}{l}\text { Percentage } \\
\text { gain }\end{array}$ \\
\hline 5 & 14.14 & \\
4 & 13.30 & 5.9 \\
3 & 11.49 & 13.6 \\
2 & 8.71 & 24.2 \\
1 & 4.95 & 43.2 \\
\hline
\end{tabular}

Four of these patients received ara-A and 1 received treatment with TFT. This indicates that both drugs are relatively safe if judged by corneal toxicity.

\section{Discussion}

Trifluorothymidine and adenine arabinoside are both very effective antiviral agents. Compared to idoxuridine they are virtually free of toxic reaction. The most striking factor in our study is that the average healing time is considerably longer than that of other similar studies. Coster et al. (1976) found the average healing time in his ara-A-treated group to be $5 \cdot 13$ days and for the TFT-treated group $5 \cdot 75$. In our study the healing time for the ara-A-treated group was 10.54 days and that for the TFT-treated group the healing time was $11 \cdot 14$ days.

One of the reasons of this striking difference in healing time could be the criteria for healing. The criterion in the study of Coster et al. (1976) was the absence of fluorescein staining of the epithelium. In our study we also included the absence of cystic or oedematous changes in the area of the previous dendritic lesion as healing criteria. These changes are often indicative of persistent viral activity. Our healing criteria necessitated also a longer treatment, which in turn was probably responsible for the absence of true recurrent dendritic ulceration.
The 7 patients that failed to heal within the 22 days' limit all had oedematous and cystic changes in the area of the previous dendrite, but these irregular areas did not stain uniformly with fluorescein. Over the lesion sporadic epithelial erosions were seen, however. After a certain period the $\frac{\partial}{\sigma}$ epithelium broke down again, and an ovoid super- $\overline{\bar{p}}$ ficial lesion with sodden oedematous borders was $\underset{\mathbb{D}}{\mathrm{O}}$ seen. In addition to these lesions there was a profoundly hypaesthetic cornea. Therefore, they were diagnosed as metaherpetic lesions.

It is true that some $20 \%$ of herpetic lesions heal $\overrightarrow{\vec{E}}$ spontaneously within a reasonably short period and $\stackrel{\omega}{\omega}$ without leaving marked corneal scarring. These 0 cases were probably also present in our patient material. As a result of this study we found the interval between the appearance of the first symp- $\overrightarrow{\dot{\omega}}$ toms and the moment treatment was started one $\omega$ of the most important parameters in the study of $\stackrel{\circ}{\triangle}$ antiviral drugs. This is very clearly demonstrated $\vec{c}$ by the flat trajectory in Fig. 2. After 4 days the relative efficacy of both TFT and ara-A is markedly diminished as indicated by the steep trajectory. This parameter is neglected in other antiviral drug $\vec{\bullet}$ studies.

We are very grateful for the preparation, coding, and donation of TFT and ara-A by the Laboratories Dr Gerhard Mann $\mathrm{GmbH}$ and Dr Thilo \& Co. GmbH, respectively.

\section{References}

Coster, D. J., McKinnon, J. R., McGill, J. I., Jones, B. R., and Fraunfelder, F. T. (1976). Clinical evaluation of adenine arabinoside and trifluorothymidine in the treatment of corneal ulcers caused by herpes simplex virus. Journal of Infective Diseases, 133, A173-A177.

Jones, B. R. (1967). Prospects in treating viral disease of the eye. Transactions of the Ophthalmological Societies of the United Kingdom, 87, 537-579.

Kaufman, H. E., Martola, E. L., and Dohlman, C. (1962). Use of 5-iode-2'-deoxyuridine (IDU) in treatment of herpes simplex keratitis. Archives of Ophthalmology, 68, 235-239.

Laibson, P. R., and Leopold, I. H. (1964). An evaluation of double-blind IDU therapy in 100 cases of herpetic keratitis. Transactions of the American Academy of Ophthalmology and Otolaringology, 68, 21-34.

Pavan-Langston, D., and Dohlman, C. (1972). A doubleblind clinical study of adenine arabinoside therapy of $N$ viral keratoconjunctivitis. American Journal of Ophthal- స్ట mology, 74, 81-88.

Wellings, P. C., Awdry, P. N., Bors, F. H., Jones, B. R., Brown, D. C., and Kaufman, H. E. (1972). Clinical $\Theta$ evaluation of trifluorothymidine in the treatment of $\overline{\mathbb{D}}$ herpes simplex corneal ulcers. American Journal of Ophthalmology, 73, 932-942. 